\title{
FACTORES QUE OPTIMIZAN LA EFECTIVIDAD DEL POLVO DE RAÍZ DE Senecio salignus CONTRA EL GORGOJO MEXICANO DEL FRIJOL
}

\section{FACTORS THAT OPTIMIZE THE EFFECTIVENESS OF THE POWDER OBTAINED FROM Senecio salignus ROOTS AGAINST THE MEXICAN BEAN WEEVIL}

\author{
Ernesto López Pérez ${ }^{1}$, Cesáreo Rodríguez Hernández ${ }^{2 *}$ y Ramón Garza García ${ }^{3}$
}

\begin{abstract}
${ }^{1}$ Desarrollo Sustentable, Unidad Yajalón, Universidad Intercultural de Chiapas. 29930, Yajalón, Chiapas, México. ${ }^{2}$ Postgrado en Fitosanidad-Entomología y Acarología, Colegio de Postgraduados, Campus Montecillo. 56230, Montecillo, Texcoco, Estado de México, México. Tel. 01-595-95-20200 ext: 1678. ${ }^{3}$ Entomología, Instituto Nacional de Investigaciones Forestales, Agrícolas y Pecuarias. 56230. Chapingo, Estado de México, México.

* Autor para correspondencia (crhernan@colpos.mx)
\end{abstract}

\section{RESUMEN}

La raíz de chilca (Senecio salignus DC., Asteraceae) ha mostrado ser efectiva para el control del gorgojo mexicano del frijol (Zabrotes subfasciatus Boheman, Coleoptera: Bruchidae). Sin embargo, falta determinar el efecto de varios factores para hacer un uso racional de este recurso natural y obtener el mejor efecto insecticida. En esta investigación se efectuaron bioensayos para conocer: a) La efectividad de raíz de $S$. salignus contra $Z$. subfasciatus a diversos tiempos de almacenamiento; b) El periodo de protección de la raíz de chilca contra esta plaga; c) El efecto del polvo de esa raíz en la oviposición de $Z$. subfasciatus en frijol (Phaseolus vulgaris L., Fabaceae) 'Flor de mayo'; y d) Efecto del polvo en granos de un frijol nativo de los Altos de Chiapas, para el control de este gorgojo. En un frasco de vidrio de $250 \mathrm{~mL}$ se colocaron $100 \mathrm{~g}$ de frijol, polvo vegetal de raíz de chilca (desde 0.05 hasta $0.5 \mathrm{~g}$ ) y 10 parejas de gorgojos. A los 4 d postinfestación se registró la mortalidad de adultos y el número de huevos del insecto por grano del frijol, y a los 50 d se cuantificó la emergencia de adultos y el grado de daño al grano. El polvo de la raíz de chilca a $0.5 \%$, colectada en cualquier fecha del año y almacenada hasta por siete meses, mató al adulto de Z. subfasciatus y protegió así al frijol almacenado, con efecto tóxico hasta los $30 \mathrm{~d}$ de postimpregnación; inhibió totalmente la oviposición de los insectos; y también el polvo fue efectivo en granos del frijol negro de mata contra este gorgojo.

Palabras clave: Senecio salignus, gorgojo mexicano del frijol, bioinsecticidas.

\section{SUMMARY}

Chilca (Senecio salignus DC., Asteraceae) root has shown effectiveness for the control of the Mexican bean weevil (Zabrotes subfasciatus Boheman, Coleoptera: Bruchidae); however, several other factors should be evaluated in order to reach a rational use of this natural resource and to obtain the best insecticidal effect. Several bioassays were carried out in order to know: a) The effecttiveness of the root of $S$. salignus collected at several dates and different storage times, against $Z$. subfasciatus; b) The period that chilca roots can protect against this insect pest; c) The effect of chilca root powder on the oviposition of $Z$. subfasciatus in bean (Phaseolus vulgaris L., Fabaceae) grains cv. 'Flor de mayo'; and d) The effect of this powder on a native black bean from Chiapas, México, also regarding the control of this weevil. Ten pairs of the Mexican bean weevil adults were placed in a glass jar which contained from 0.05 to $0.5 \mathrm{~g}$ of ground chilca root and $100 \mathrm{~g}$ of bean grains. At 4 and $50 \mathrm{~d}$ after infestation, insect mortality and number of eggs per grain were registered, along with emergence of new adults and level of damage to the bean grains. The powder of chilca root at $0.5 \%$ ( $5 \mathrm{~g}$ of powder per $\mathrm{kg}$ of bean grains), collected at any time of the year and stored up to seven months, was able to kill the insect adults of $Z$. subfasciatus and thus to protect the stored bean up to $30 \mathrm{~d}$ after impregnation. The chilca powder also inhibited the insect oviposition, and was effective against the pest on a native black bean.

Index words: Senecio salignus, Mexican bean weevil, bioinsecticides.

\section{INTRODUCCIÓN}

Las plantas son organismos vivos que están en constante interacción con su ecosistema, de manera que los metabolitos secundarios que producen están cambiando continuamente de concentración. Esto hace que las plantas, sus extractos o sustancias, algunas veces no manifiesten actividad biológica mientras que en otras ocasiones muestran actividad insectistática o insecticida. Esta variabilidad indica que existen factores intrínsecos y extrínsecos que afectan la concentración y actividad de las sustancias insectistáticas e insecticidas. Los factores como edad de la planta (White, 1948), temperatura y humedad relativa atmosférica (Singh, 1987), precipitación (Schmutterer, 1990), fertilidad y $\mathrm{pH}$ del suelo donde crece la planta (Ermel et al., 1987), intensidad de la luz solar (Stokes y Redfern, 1982), humedad relativa y temperatura del almacén (Ermel et al., 1987), y del ambiente durante el bioensayo (Harries, 1945), deben estar en condiciones óptimas para alcanzar el mejor efecto en insectos. 
En la chilca (Senecio salignus DC., Asteraceae), una planta con potencial insecticida para el gorgojo mexicano del frijol (Zabrotes subfasciatus Boheman, Coleoptera: Bruchidae), se ha detectado que la biomasa seca y pulverizada de raíz actúa mejor que la de hoja y tallo (Rodríguez y López, 2001), y que la concentración más adecuada del polvo es $0.25 \%$ (0.25 g de polvo por $100 \mathrm{~g}$ de grano de frijol), el cual actúa por contacto y su mejor tamaño de partícula es $<0.25 \mathrm{~mm}$ de diámetro (López et $a l ., 2007)$, además hay otros factores que deben evaluarse para el mejor aprovechamiento racional de este recurso.

Los objetivos de este trabajo fueron determinar la efectividad de la raíz de chilca en función de la fecha de colecta, tiempo de almacenamiento y persistencia, en cuanto a la oviposición de Z. subfasciatus en los granos de dos variedades de frijol (Phaseolus vulgaris L., Fabaceae) y el control de esta plaga.

\section{MATERIALES Y MÉTODOS}

\section{Colecta de raíz de chilca}

La raíz de chilca se colectó en Amatenango del Valle, Chiapas, México, $\left(16^{\circ} 32^{\prime} \mathrm{LN}, 92^{\circ}\right.$ 26' $^{\prime} \mathrm{LO}, 1810$ $\mathrm{msnm}$ ), en abril y octubre del 2004 y en enero, abril y junio del 2005 ( $3 \mathrm{~kg}$ de raíz fresca en cada fecha), las que se identificaron como colectas 1 a 5 . Las muestras de raíz se secaron a la sombra por $40 \mathrm{~d}$ y se mantuvieron en cajas de cartón sin exposición a rayos solares; $8 \mathrm{~d}$ antes de realizar los bioensayos, la raíz se pulverizó con un molino manual hasta obtener partículas con diámetro $<0.25$ $\mathrm{mm}$. Las muestras de polvo se almacenaron en bolsas de polietileno a temperatura ambiente, en Texcoco, Edo. de México (19 ${ }^{\circ} 29^{\prime} \mathrm{LN}, 98^{\circ}$ 54', $\left.2250 \mathrm{msnm}\right)$. La determinación taxonómica de la chilca fue realizada por el M. C. Ricardo Vega Muñoz, Curador del HerbarioHortorio, del Programa de Botánica del Colegio de Postgraduados-Campus Montecillo.

\section{Cría del gorgojo}

En un frasco de vidrio de $2 \mathrm{~L}$ se colocó $1 \mathrm{~kg}$ del frijol var. 'Flor de mayo' que previamente se limpió y seleccionó para eliminar piedras, otras semillas y granos quebrados, anormales y pequeños; luego se lavó (para eliminar polvo y residuos de químicos) y se almacenó a $-4{ }^{\circ} \mathrm{C}$ por $20 \mathrm{~d}$ para eliminar una posible infestación insectil. El grano así pretratado y descongelado, se infestó con aproximadamente 3000 adultos de $Z$. subfasciatus, en una proporción sexual 1:1, obtenidos de la cría existente en el laboratorio. Los insectos se mantuvieron en confinamiento en el frasco durante $3 \mathrm{~d}$ para que copularan y ovipositaran, tiempo que fue suficiente para regular el desarrollo de la primera generación, y para uniformizar los tiempos de emergencia de los adultos. A los 30 d postinfestación se seleccionaron los adultos de la primera generación a ser usados para infestar otros frascos con grano. Este proceso de cría se hizo en forma periódica para incrementar la población en todos los estados biológicos y obtener adultos en cantidad suficiente para llevar a cabo los bioensayos. La cría del gorgojo se mantuvo en una cámara bioclimática con temperatura de $27 \pm 2{ }^{\circ} \mathrm{C}$, humedad relativa de $50 \pm 5 \%$ y un fotoperiodo de 12:12 $\mathrm{h}$ de luz:oscuridad.

\section{Bioensayos}

Para evaluar la efectividad del bioinsecticida, en un frasco de vidrio de $250 \mathrm{~mL}$ se colocaron $100 \mathrm{~g}$ del frijol var. 'Flor de mayo', y se mezclaron homogéneamente con $0.05,0.075,0.10$ y $0.5 \mathrm{~g}$ de polvo de raíz de chilca. Posteriormente, el frijol se infestó con 10 parejas de gorgojos de $Z$. subfasciatus con menos de $24 \mathrm{~h}$ de edad. Esto se hizo en cinco repeticiones por cada concentración de polvo, más un testigo que consistió de granos de frijol no tratado, para un total de cinco tratamientos.

El efecto de la fecha de colecta y el tiempo de almacenamiento de raíz de chilca se determinó mediante tres experimentos. En el primero se evaluó el polvo de la raíz de las colectas 1,2 y 3 que tenían 12, 6 y 3 meses de almacenamiento (mda), respectivamente; en el segundo ensayo se probó raíz de las colectas 2, 3, 4 y 5 con 10, 7, 4 y 2 mda, respectivamente; y en el tercero se empleó raíz de las colectas $1,2,3,4$ y 5 con 18, 12, 9, 6 y 4 mda, respectivamente. En un cuarto bioensayo se evaluó el efecto protectivo del polvo de chilca a $0.5 \%$ de concentración, en granos de frijol infestados con el gorgojo, a diferentes tiempos de postimpregnación: 0, 8, $15,22,30,60,90,120,150$ y 180 d. La mortalidad de adultos infestantes y la emergencia de adultos en la primera generación se registraron a los 4 y 50 d después de la infestación. El daño del grano picado en los tres primeros experimentos se registró a los 50 d de postratamiento. La mortalidad registrada en estos bioensayos se corrigió con la observada en el testigo, por medio de la ecuación de Abbott (1925), y cuando ésta excedió de $12 \%$ el experimento se repitió.

En otros dos bioensayos (5 y 6) en los que se usó la misma metodología, se evaluaron diferentes concentraciones de polvo de chilca: 5) $0.05,0.075,0.1$ y $0.5 \%$, para cuantificar a los $4 \mathrm{~d}$ después de la incorporación de los gorgojos, el número de huevos en 20 granos del frijol 'Frijol de mayo' seleccionados al azar en cada repetición; y 6) $0.05,0.1$ y $0.5 \%$, en granos del frijol nativo de los Altos de Chiapas, de color negro y de mata. En estos dos últimos ensayos la mortalidad de insectos adultos se 
registró a los 4 d, y la emergencia de nuevos adultos en la primera generación y la cantidad de granos dañados se hizo a los 50 d después de la infestación. En los tres últimos bioensayos $(4,5$ y 6$)$ se usó raíz de la colecta 3 , con 4 a 5 mda.

\section{Análisis estadístico}

Los datos de mortalidad de insectos adultos, emergencia de nuevos adultos, granos dañados y número de huevos de estos experimentos se analizó mediante un diseño completamente al azar, y se sometieron a la prueba de supuestos de normalidad de errores (cuantiles) y de homogeneidad de varianza (prueba de Bartlett). Después se les aplicó la prueba de comparaciones múltiples de Kruskal-Wallis para elegir al mejor tratamiento $(\alpha=0.05)$. Todos los análisis se llevaron a cabo con el programa estadístico SAS (SAS Institute, 1999).

\section{RESULTADOS Y DISCUSIÓN}

\section{Fechas de colecta y tiempo de almacenamiento en la efectividad de la raíz de chilca}

Las fechas de colecta de la raíz de chilca y tiempos de almacenamiento de su polvo, difirieron $(\mathrm{P} \leq 0.05)$ en su efecto sobre la mortalidad en adultos (desde 11 hasta 100 $\%)$ y sobre la inhibición de emergencia de adultos en la primera generación de Z. subfasciatus (desde 18.6 hasta $100 \%)$, en la dosis de $0.5 \%(\mathrm{p} / \mathrm{p})$, y por tanto variaron en el nivel de protección al grano del frijol 'Flor de mayo' desde 90 hasta 100 \% (Cuadro 1). Las raíces de las colectas 2, 3, 4 y 5 con tiempo de almacenamiento desde 2 hasta 7 meses, ocasionaron $100 \%$ de mortalidad, inhibieron completamente la emergencia de adultos y evitaron el daño al grano, en tanto que las raíces de las colectas 1,2 y 3 con tiempo de almacenamiento desde 9 hasta 18 meses provocaron mortalidad desde 97 hasta 11 $\%$, con emergencia de adultos desde 0 hasta $81 \%$ y con daños al grano desde 0 hasta $30 \%$.

Los resultados de estos bioensayos permiten inferir que para eliminar totalmente los gorgojos, la raíz de chilca puede colectarse en cualquier fecha del año pero no ser almacenada por más de 7 meses, ya que después de este tiempo su efectividad insecticida comienza a decrecer, de forma que a los 18 mda pierde su capacidad para proteger al grano de frijol almacenado. A los $9 \mathrm{mda}$, no obstante que la raíz de la colecta 3 inhibió completamente la emergencia de adultos y evitó totalmente el daño al grano, el polvo perdió $3 \%$ de efectividad, lo que representa riesgos en la protección del grano de frijol al transcurrir el tiempo de almacenamiento.
La raíz de chilca colectada en diferentes estaciones del año y almacenada por 7 o menos meses mostró $100 \%$ de efectividad, lo que denota que la toxicidad en campo no fluctuó sustancialmente con los cambios estacionales y climáticos del año, probablemente por ser una planta perenne resistente a heladas (Jones, 1979).

La raíz de chilca con 3 mda y aplicada a $0.5 \%$ mostró efectividad similar a la reportada por Rodríguez y López (2001), quienes obtuvieron $100 \%$ de mortalidad, $0 \%$ de emergencia de adultos de Z. subfasciatus y $0 \%$ de daño al grano. A esta misma concentración fue mejor que la raíz de chilca almacenada por 14 meses (Rodríguez y Rosas, 2002), y que en el gorgojo del maíz (Sitophilus zeamais Motschulsky, Coleoptera: Curculionidae) causó $93 \%$ de mortalidad, $9.6 \%$ de emergencia de adultos y $1.8 \%$ de daño al grano. Se confirmó así que la raíz de la chilca con más de siete meses de almacenamiento no puede eliminar a toda la población de gorgojos ni proteger completamente al grano.

La raíz de chilca secada y almacenada en sombra y a temperatura ambiente de una región con clima templado, mostró alto potencial para el control de Z. subfasciatus, aunque conviene tener cuidado con las condiciones de almacenamiento de la raíz, para evitar exponerla a la luz solar o alta temperatura $\left(>45{ }^{\circ} \mathrm{C}\right)$ porque su toxicidad puede disminuir. Al respecto se ha reportado que el contenido de azadiractina en los frutos del nim (Azadirachta indica A. Juss., Meliaceae) puede bajar en $20 \%$ al incrementar la temperatura de almacenamiento de 20 a $70{ }^{\circ} \mathrm{C}$ (Stokes y Redfern, 1982; Ermel et al., 1987).

\section{Efectividad del polvo de raíz de chilca}

El polvo de la raíz de chilca a $0.5 \%$ mató al total de adultos de $Z$. subfasciatus en los primeros $30 \mathrm{~d}$ postaplicación (Cuadro 2). Después de este tiempo decreció su efectividad ya que a los 60 d se observó una disminución de $5 \%$ en su efecto, y a los 150 y 180 d su toxicidad disminuyó drásticamente porque hubo sobrevivencia de 43 y $45 \%$ de Z. subfasciatus, respectivamente. Respecto a la emergencia de adultos en la primera generación, el polvo de raíz de chilca inhibió por completó (100 \%) la emergencia de gorgojos en los primeros $30 \mathrm{~d}$, mientras que a los 60 y $90 \mathrm{~d}$ ésta fue del $99.7 \%$. A partir de los $120 \mathrm{~d}$ del tratamiento, la emergencia de insectos empezó a aumentar de manera que a los $180 \mathrm{~d}$ hubo emergencia de una tercera parte de la población de adultos, comparado con el testigo. 
Cuadro 1. Mortalidad, emergencia y daño de $Z$. subfasciatus en frijol 'Flor de mayo' tratado con polvo de raíz de chilca a $0.5 \%$ (p/p), colectada en diferentes fechas y con diversos tiempos de almacenamiento.

\begin{tabular}{|c|c|c|c|c|c|c|c|}
\hline $\begin{array}{l}\text { Fecha de } \\
\text { colecta }\end{array}$ & Abr-04 & Oct-04 & Ene-05 & Abr-05 & Jun-05 & Testigo & $\mathrm{X}^{2}$ \\
\hline Mortalid. (\%) & $3 \pm 1.5^{\llbracket}(50)^{\dagger} \mathrm{b}$ & $10.5 \pm 0(100) \mathrm{a}$ & $10.5 \pm 0(100) \mathrm{a}$ & & & & 0.95 \\
\hline Daño (\%) & $12 \pm 3.5(5.3) b$ & $6 \pm 0(0.0) c$ & $6 \pm 0(0.0) c$ & & & $18 \pm 1.5(36.4) \mathrm{a}$ & 0.89 \\
\hline T.a. (m) & & 10 & 7 & 4 & 2 & & \\
\hline Mortalid. (\%) & & $3 \pm 1.5(83) b$ & $13 \pm 0(100) \mathrm{a}$ & $13 \pm 0(100) a$ & $13 \pm 0(100) a$ & & 0.97 \\
\hline T.a. (m) & 18 & 12 & 9 & 6 & 4 & & \\
\hline Mortalid. (\%) & $3 \pm 1.1(11) \mathrm{d}$ & $8 \pm 1.5(75) c$ & $16 \pm 4.1(97) b$ & $19 \pm 0(100) \mathrm{a}$ & $19 \pm 0(100) \mathrm{a}$ & & 0.92 \\
\hline Emergen. (\%) & $24 \pm 3.5(81.4) b$ & $18 \pm 1.5(22.3) \mathrm{c}$ & $8 \pm 0(0.0) d$ & $8 \pm 0(0.0) d$ & $8 \pm 0(0.0) d$ & $27 \pm 0(100) \mathrm{a}$ & 0.96 \\
\hline Daño $(\%)$ & $25 \pm 3.3(30.9) \mathrm{a}$ & $18 \pm 1.5(9.96) \mathrm{b}$ & $8 \pm 0(0.0) \mathrm{c}$ & $8 \pm 0(0.0) \mathrm{c}$ & $8 \pm 0(0.0) \mathrm{c}$ & $26 \pm 2.9(34.5) \mathrm{a}$ & 0.95 \\
\hline
\end{tabular}

Medias con letras iguales entre las hileras no son estadísticamente diferentes (Kruskal-Wallis, 0.05); T.a. = tiempo de almacenamiento; $\mathrm{m}=$ meses; promedios y desviaciones estándar (datos observados, entre paréntesis).

Cuadro 2. Mortalidad y emergencia de adultos de $Z$. subfasciatus en 'Frijol flor' de mayo tratado con polvo de raíz de chilca a $0.5 \%$, en diferentes tiempos de infestación.

\begin{tabular}{lccccc}
\hline $\begin{array}{l}\text { T.i. } \\
(\mathrm{d})\end{array}$ & $\begin{array}{c}\text { Mortalidad } \\
(\%)\end{array}$ & $\begin{array}{c}\text { Emergencia } \\
(\%)\end{array}$ & $\begin{array}{c}\text { T.i. } \\
(\mathrm{d})\end{array}$ & $\begin{array}{c}\text { Mortalidad } \\
(\%)\end{array}$ & $\begin{array}{c}\text { Emergencia } \\
(\%)\end{array}$ \\
\hline 0 & $37 \pm 0(100) \mathrm{a}$ & $17 \pm 0(0.0) \mathrm{c}$ & 60 & $26.5 \pm 10.1(95) \mathrm{b}$ & $24 \pm 9.5(0.3) \mathrm{c}$ \\
8 & $37 \pm 0(100) \mathrm{a}$ & $17 \pm 0(0.0) \mathrm{c}$ & 90 & $18.8 \pm 1.7(91) \mathrm{c}$ & $20 \pm 8.4(0.3) \mathrm{c}$ \\
15 & $37 \pm 0(100) \mathrm{a}$ & $17 \pm 0(0.0) \mathrm{c}$ & 120 & $13.7 \pm 2.7(80.7) \mathrm{d}$ & $40 \pm 2.9(9.6) \mathrm{ab}$ \\
22 & $37 \pm 0(100) \mathrm{a}$ & $17 \pm 0(0.0) \mathrm{c}$ & 150 & $5.8 \pm 1.7(57) \mathrm{e}$ & $36 \pm 11.3(8.0) \mathrm{b}$ \\
30 & $37 \pm 0(100) \mathrm{a}$ & $17 \pm 0(0.0) \mathrm{c}$ & 180 & $5.2 \pm 4.0(55) \mathrm{e}$ & $48 \pm 1.5(31.7) \mathrm{a}$ \\
$\mathrm{X}^{2}$ & & & & 0.94 & 0.83 \\
\hline
\end{tabular}

Medias en los tratamientos de mortalidad y emergencia con letras iguales no son estadísticamente diferentes (Kruskal-Wallis, 0.05); T.i. = tiempo de infestación; promedios y desviaciones estándar (datos observados, entre paréntesis).

Con estos resultados se deduce que los adultos de $Z$. subfasciatus que se encuentren o lleguen al frijol almacenado en los primeros $30 \mathrm{~d}$ posteriores al tratamiento con el polvo de raíz de chilca a $0.5 \%$, morirán sin dejar descendientes; en cambio; los gorgojos que arriben después de un mes de haberse tratado el frijol, podrían sobrevivir en $5 \%$.

La efectividad de la chilca disminuyó en $45 \%$ a los 6 meses de impregnación; sin embargo, en una prueba adicional (datos no mostrados) se corroboró que al volver a mezclar el polvo con el frijol e infestar con adultos de Z. subfasciatus a los 180 d, la efectividad sólo disminuyó en $13 \%$, lo que indica que a partir del segundo mes la baja en toxicidad se sebe en parte, a la pérdida de la capacidad de adhesión al grano, porque con el tiempo el polvo se acumula en el fondo del frasco y el insecto no entra en contacto con éste. Por ello, es necesario voltear el costal, bolsa o recipiente de almacenamiento del grano a los $30 \mathrm{~d}$, para así incrementar la vida útil del polvo al permitir que el grano se impregne nuevamente.

La protección del polvo de raíz de chilca a $0.5 \%$ por $30 \mathrm{~d}$ contra $Z$. subfasciatus del presente trabajo fue 25 y
$33 \%$, con respecto al tiempo de persistencia de los polvos de anona blanca (Annona squamosa L., Annonaceae) y pimienta negra (Piper nigrum L., Piperaceae) a $1 \%$ aplicados en frijol judea (Vigna radiata L.) para controlar al gorgojo graham (Callosobruchus analis F., Coleoptera: Bruchidae) (Junepa y Patel, 2002). Es de suponer que el periodo de protección con chilca puede incrementarse si se aumenta su concentración a 1 $\%$. Con el aceite de nim se ha observado que al aumentar la cantidad de 2.5 a $7.5 \mathrm{~mL} \mathrm{~kg}^{-1}$ de frijol, el tiempo de protección contra $Z$. subfasciatus se aumenta hasta en 4 meses (Chinwada y Giga, 1993), y que al incrementar la dosis a $10 \mathrm{~mL}$ de aceite por $\mathrm{kg}$ de grano se protege a éste hasta por 6 meses contra el gorgojo chino (Callosobruchus chinensis L.) (Singh y Sharma, 2003).

En las condiciones experimentales de este estudio (27 $\pm 2{ }^{\circ} \mathrm{C}, 50 \pm 5 \%$ de humedad relativa y $12 \mathrm{~h}$ de escotofase), el polvo de raíz de chilca controló satisfactoriamente el ataque de Z. subfasciatus hasta $30 \mathrm{~d}$ después de su aplicación. Sin embargo, en otros experimentos se ha reportado que la actividad insecticida de otras plantas es afectada por la temperatura; por ejemplo, el aceite de piretro (Tanacetum cinerariifolium 
(Trevir.) Sch. Bip., Asteraceae) provocó 65 y $38 \%$ de mortalidad en la chicharrita del betabel (Eutettix tenellus Baker, Hemiptera: Cicadellidae) en condiciones de 21.12 y $32.24{ }^{\circ} \mathrm{C}$, respectivamente (Harries, 1945) Este efecto del calor podría también suceder en la chilca.

\section{Efecto de la concentración del polvo de raíz de chilca en la oviposición}

La raíz de chilca pudo empezar a inhibir la oviposición de Z. subfasciatus a partir de la concentración de 0.075 $\%$, y la inhibió completamente con $0.5 \%$ (Cuadro 3). Las concentraciones iguales o mayores a $0.075 \%$ incrementaron el número y porción de granos cubiertos con polvo, lo que dificultó al insecto hembra encontrar espacios adecuados para ovipositar, y lo obligó a recorrer otros granos e impregnarse con el polvo, lo que finalmente le ocasionó la muerte o le impidió realizar sus funciones fisiológicas de manera normal.

\begin{tabular}{|c|c|}
\hline Concentración (\%) & Huevos por grano \\
\hline 0.50 & $5 \pm 0(0.00)^{\dagger} \mathrm{c}$ \\
\hline 0.10 & $7.9 \pm 3.8(0.06) b c$ \\
\hline 0.075 & $11.1 \pm 3.8(0.16) b$ \\
\hline 0.05 & $19.2 \pm 3.2(1.22) \mathrm{a}$ \\
\hline 0.0 & $21.8 \pm 2.3(1.90) \mathrm{a}$ \\
\hline $\mathrm{X}^{2}$ & 0.84 \\
\hline
\end{tabular}

La inhibición total de la oviposición con la concentración de $0.5 \%$ se debe a que los adultos de $1 \mathrm{~d}$ de edad mueren al segundo día de exposición, como también observaron López et al. (2007); por tanto, los insectos no alcanzan a llegar al cuarto día, que es el tiempo de mayor oviposición de esta especie (Golob y Kilminster, 1982).

\section{Efecto del polvo de raíz de chilca en el frijol negro nativo de los Altos de Chiapas}

El polvo de raíz de chilca a $0.5 \%$ de concentración mató a todos los individuos de Z subfasciatus y protegió así al grano del frijol negro nativo de los Altos de Chiapas; a menores concentraciones disminuyó su efectividad (Cuadro 4). La mortalidad obtenida en este experimento fue similar a la encontrada por Rodríguez y López (2001) y López et al. (2007) con polvo de raíz de chilca a $0.5 \%$, aplicado en el frijol 'Flor de mayo'. Esto indica que el frijol negro de mata, con aparente resistencia (en pruebas preliminares) por ser poco dañado y menos preferido para refugio de insectos adultos y para oviposición, no mostró efecto aditivo con la raíz de chilca para el control de Z. subfasciatus.

Según Mazzonetto y Vendramim (2004) ${ }^{1}$, no existe interacción positiva entre polvo de epazote (Chenopodium ambrosioides L., Amaranthaceae), que causó $100 \%$ de mortalidad a la concentración de $3 \%$, y el nivel de resistencia del grano en cuanto al control del gorgojo pardo del frijol (Acanthoscelides obtectus (Say), Coleoptera: Bruchidae).

Con esta investigación no solamente se detectó efectividad de la raíz de chilca en el frijol nativo negro de mata, sino también se definieron otros aspectos que permiten optimizar el uso de la chilca contra $Z$. subfasciatus. Al respecto, se determinó que la raíz se puede colectar en cualquier fecha del año pero no debe ser guardada por más de 7 meses, el grano debe reimpregnarse cada $30 \mathrm{~d}$ si se almacena por mayores periodos, y que el polvo de la raíz debe aplicarse en dosis de $5 \mathrm{~kg}$ por tonelada de frijol almacenado para lograr así inhibir completamente la oviposición y evitar la proliferación de la plaga. Estas consideraciones se suman a las aportadas por Rodríguez y López (2001), quienes recomendaron utilizar el polvo de raíz y no el de hoja ni de tallo, así como a las de López et al. (2007) quienes señalaron que el polvo de la raíz actúa por contacto y manifiesta su mayor actividad con partículas menores de $0.25 \mathrm{~mm}$ de diámetro.

La chilca, que se distribuye desde el sur de los Estados Unidos hasta Honduras, es una planta medicinal que incrementa su importancia económica con estas propiedades insecticidas, por lo que debe procurarse su conservación como especie; entre las medidas protectiva, sería no extraer la raíz sino cortar tallos y enraizarlos para así disponer de suficiente material que permita su aprovechamiento en pequeña o gran escala como plaguicida natural. Romo de Vivar et al. (2007) reportaron la presencia de alcaloides en esta especie, lo que conlleva el riesgo de un efecto tóxico en el hombre y en los animales domésticos; para evitar ese riesgo, y considerando que el polvo no penetra en el grano sino que se precipita al fondo del costal o recipiente donde se almacena la semilla, y que la dosis es relativamente pequeña ( $5 \mathrm{~kg}$ de polvo por tonelada de grano), se recomienda tamizar el grano o lavarlo antes de consumirlo.

\footnotetext{
${ }^{1}$ Mazzonetto F, J D Vendramim (2004) "Efecto asociado de genotipos resistentes y polvos vegetales sobre Acanthoscelides obtectus en frijol armazenado. XX Congresso Brasileiro de Entomologia. 5 a 10 setembro de 2004. Gramado, RS, Brasil.
} 
Cuadro 4. Mortalidad, emergencia y daño de $Z$. subfasciatus en frijol negro de mata nativo de los Altos de Chiapas, tratado con diferentes concentraciones de polvo de raíz de $S$. salignus.

\begin{tabular}{|c|c|c|c|}
\hline Concentración (\%) & Mortalidad (\%) & Emergencia (\%) & Daño (\%) \\
\hline 0.50 & $18 \pm 0(100) \mathrm{a}$ & $3.5 \pm 0(0.0) \mathrm{d}$ & $3.5 \pm 0(0.0) \mathrm{d}$ \\
\hline 0.10 & $13 \pm 1.5(89.0) b$ & $8.1 \pm 3.2(2.5) \mathrm{c}$ & $7.9 \pm 2.9(0.6) \mathrm{c}$ \\
\hline 0.05 & $8 \pm 1.5(48.0) \mathrm{c}$ & $12.4 \pm 2.4(15.3) b$ & $12.6 \pm 2.3(3.5) b$ \\
\hline 0.0 & $3 \pm 0(0.0) d$ & $18 \pm 0(100) \mathrm{a}$ & $18 \pm 1.5(35.6) \mathrm{a}$ \\
\hline $\mathrm{X}^{2}$ & 0.97 & 0.89 & 0.89 \\
\hline
\end{tabular}

De esta forma, la chilca que ha entrado en desuso en muchas partes de la República Mexicana por ese riesgo, podría utilizarse para la protección del frijol almacenado en las zonas agrícolas de subsistencia, como una medida económica de control de gorgojos y constituir una alternativa orgánica de conservación de granos.

\section{CONCLUSIONES}

La raíz de chilca ( $S$. salignus L.), colectada en cualquier fecha del año y hasta con 7 meses de almacenamiento e impregnada como polvo a una concentración de $0.5 \%$ ( $5 \mathrm{~kg}$ de polvo por tonelada de grano) al grano de frijol, a nivel de laboratorio es capaz de matar todos los adultos del gorgojo mexicano del frijol ( $Z$. subfasciatus), y así puede proteger completamente al frijol almacenado hasta por $30 \mathrm{~d}$ e inhibir totalmente la oviposición.

\section{BIBLIOGRAFÍA}

Abbott W S (1925) A method for computing the effectiveness of the insecticide. J. Econ. Entomol. 18:265-267.

Chinwada P, D Giga (1993) Vegetable and neem oils as protectants of stored beans against bruchids. CIAT African Workshop Series 25:40-49.

Ermel K, E Pahlich, H Schmutterer (1987) Azadirachtin content of neem kernels from different geographical locations, and its dependence on temperature, relative humidity, and light. In: Natural Pesticides From the Neem Tree (Azadirachta indica A. Juss) and Other Tropical Plants. H Schmutterer, K R S Ascher (eds). GTZ. Eschborn, Germany. pp:171-184.

Golob P, A Kilminster (1982) The biology and control of Zabrotes subfasciatus Boheman (Coleoptera: Bruchidae). J. Stored Prod. Res. 18:95-101.

Harries F H (1945) Some factors affecting the insecticidal action of pyrethrum extracts on the beet leafhopper. J. Econ. Entomol. 71:553-565.

Jones W (1979) Effects of 1978 freeze on native plants of Sonora, México. Des. Plants 1:33-36.

Junepa R P, J R Patel (2002) Persistence of botanical materials as protectant of green gram (Vigna radiata (L.) Wilczek) against pulse beetle (Callosobruchus analis Fabricus). Seed Res. 30:294-297.

López P E, C Rodríguez H, L D Ortega A, R Garza G (2007) Actividad biológica de la raíz de Senecio salignus contra Zabrotes subfasciatus en frijol almacenado. Agrociencia 41:95102.
Rodríguez H C, C Rosas V (2002) Actividad del epazote Teloxys ambrosioides (Chenopodiaceae) en el gorgojo del maíz Sitophilus zeamais (Coleoptera: Curculionidae). Entomol. Mex. 1:323-328.

Rodríguez H C, E López P (2001) Actividad insecticida e insectistática de la chilca (Senecio salignus) sobre Zabrotes subfasciatus. Manejo Integr. Plagas 59:19-26.

Romo de Vivar A, A Pérez-Castorena, A Arciniegas, J L Villaseñor (2007) Secondary metabolites from Mexican species of the tribe Senecioneae (Asteraceae). J. Mex. Chem. Soc. 51:160-172.

SAS Institute (1999) The SAS system for windows. Release 8. Cary, North Carolina, USA. 1643 p.

Schmutterer H (1990) Properties and potential of natural pesticides from the neem tree, Azadirachta indica. Ann. Rev. Entomol. 35:271-297.

Singh R P (1987) Comparison of antifeedant efficacy and extract yields from different parts and ecotypes of neem (Azadirachta indica A. Juss) trees. In: Natural Pesticides From the Neem Tree (Azadirachta indica A. Juss) and Other Tropical Plants. H Schmutterer, K R S Ascher (eds). GTZ. Eschborn, Germany. pp:185-194.

Singh S, G Sharma (2003) Efficacy of different oils as grain protectant against Callosobruchus chinensis in greengram and their effect on seed germination. Indian J. Entomol. 65:500-505.

Stokes J B, R E Redfern (1982) Effect of sunlight on azadirachtin: antifeeding potency. J. Env. Sci. Health 17:57-65.

White D G (1948) Production of Derris elliptica in relation to type of cutting and age at harvest. J. Econ. Entomol. 77:13-24. 